\title{
Osimertinib in combination with bevacizumab in EGFR-Mutated NSCLC with leptomeningeal metastases
}

\author{
Tao Jiang ${ }^{1,2 \#}$, Xiaobo $\mathrm{Xu}^{2 \#}$, Xiaojuan Chen ${ }^{3 \#}$, Ning Ding ${ }^{2 \#}$, Qin Hu ${ }^{4 \#}$, Caicun Zhou ${ }^{1}$, Jie $\mathrm{Hu}^{2}$ \\ ${ }^{1}$ Department of Oncology, Shanghai Pulmonary Hospital, Tongji University School of Medicine, Shanghai, China; ${ }^{2}$ Department of Pulmonary \\ Medicine, Shanghai Respiratory Research Institute, Zhongshan Hospital Affiliated to Fudan University, Shanghai, China; ${ }^{3}$ Department of \\ Gastroenterology, Wenzhou People’s Hospital, The Wenzhou Third Clinical Institute Affiliated To Wenzhou Medical University, Wenzhou, China; \\ ${ }^{4}$ Department of Pathology, Shanghai Respiratory Research Institute, Zhongshan Hospital, Fudan University, Shanghai, China \\ \#These authors contributed equally to this work. \\ Correspondence to: Dr. Jie Hu, MD, PhD. Department of Pulmonary Medicine, Shanghai Respiratory Research Institute, Zhongshan Hospital \\ Affiliated to Fudan University, No. 180, Fenglin Road, Shanghai 200032, China. Email: hujie73@126.com.
}

Submitted Aug 27, 2020. Accepted for publication Nov 26, 2020.

doi: $10.21037 /$ tlcr-20-984

View this article at: http://dx.doi.org/10.21037/tlcr-20-984

Leptomeningeal metastases (LM) occur in approximately $5-15 \%$ of non-small-cell lung cancer (NSCLC) patients with $E G F R$ mutations together with very poor prognosis, and there is no broadly accepted standard treatment (1). Although new-generation EGFR-TKIs have significantly prolonged both progression-free survival (PFS) and overall survival (OS) in patients with EGFR-mutated NSCLC $(2,3)$, little is known about the efficacy of novel EGFRTKIs (e.g., osimertinib) for patients with LM. Recently, Lee $e t$ al. compared the clinical outcomes of patients with EGFR-mutated NSCLC and LM received osimertinib with those not receive osimertinib in the Fournal of Thoracic Oncology (4). Their data showed that patients with LM received osimertinib had significantly longer OS than those not treated with osimertinib (17.0 vs. 5.5 months, $\mathrm{P}<0.001$ ), which suggested that osimertinib would be a good therapeutic option for patients with EGFR-mutated NSCLC and LM. Moreover, two recent publications suggested that combining EGFR-TKIs and bevacizumab may be efficacious for brain metastases (BMs) and protective against central nervous system (CNS) progression $(5,6)$. Herein, we presented one case to further investigate the efficacy and tolerability of osimertinib plus bevacizumab for patient with EGFR-mutated NSCLC and cytologically proven LM.

This patient was a 47 -year-old male smoker consulted our hospital with 3-week history of cough and chest distress in September 2019. The thoracic computed tomography
(CT) scan found a mass in the lower lobe of the right lung (Figure 1A). Multiple BMs and abnormal signal on meninges of cerebellum were seen in magnetic resonance imaging (MRI) scan, but ${ }^{18} \mathrm{~F}$-deoxyglucose on positron emission tomography (PET) scan did not find other distant metastases. Bronchoscopy biopsy and cytology of cerebrospinal fluid (CSF) revealed lung adenocarcinoma (Figure $1 B$ ). Subsequent genotype of primary lesion revealed EGFR exon 19 deletion, which was confirmed by targeted next-generation sequencing (NGS) panel of 139 genes (PULMOCAN, GENESEEQ Technology Inc., Nanjing, China) of CSF cytologic specimen (Figure 1B). The patients suffered headache and violent vomiting during hospitalization. Then he received osimertinib $(80 \mathrm{mg}$, once daily) plus bevacizumab $(7.5 \mathrm{mg} / \mathrm{kg}, 21$-day cycle) as the first-line treatment from October 2, 2019. Strikingly, the abnormal signal on LM disappeared (Figure 1A) and his LM-related symptoms significantly improved. A marked shrinkage of both primary lesion and BMs was also observed after 6 weeks of osimertinib plus bevacizumab treatment (Figure 1A). Moreover, the efficacy has been maintained more than 10 months (last follow-up: August 8, 2020). This regimen was well tolerated, with only grade 2 skin rash and grade 1 hypertension during regular follow-up.

In previous study, 22 NSCLC patients (previously received EGFR-TKIs, from the AURA program) with EGFR T790M mutation and radiologically-detected LMs were treated with osimertinib ( $80 \mathrm{mg}$ once daily). LM ORR 

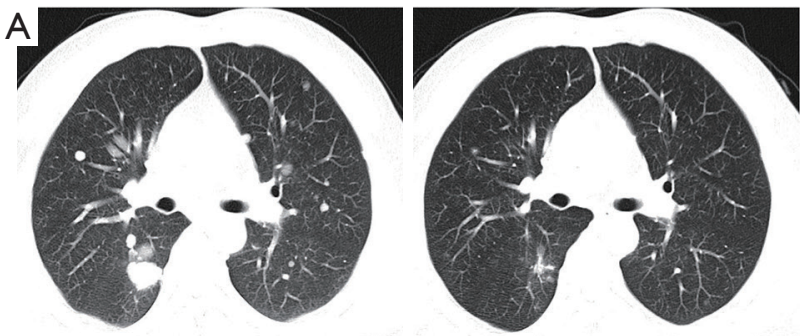

B
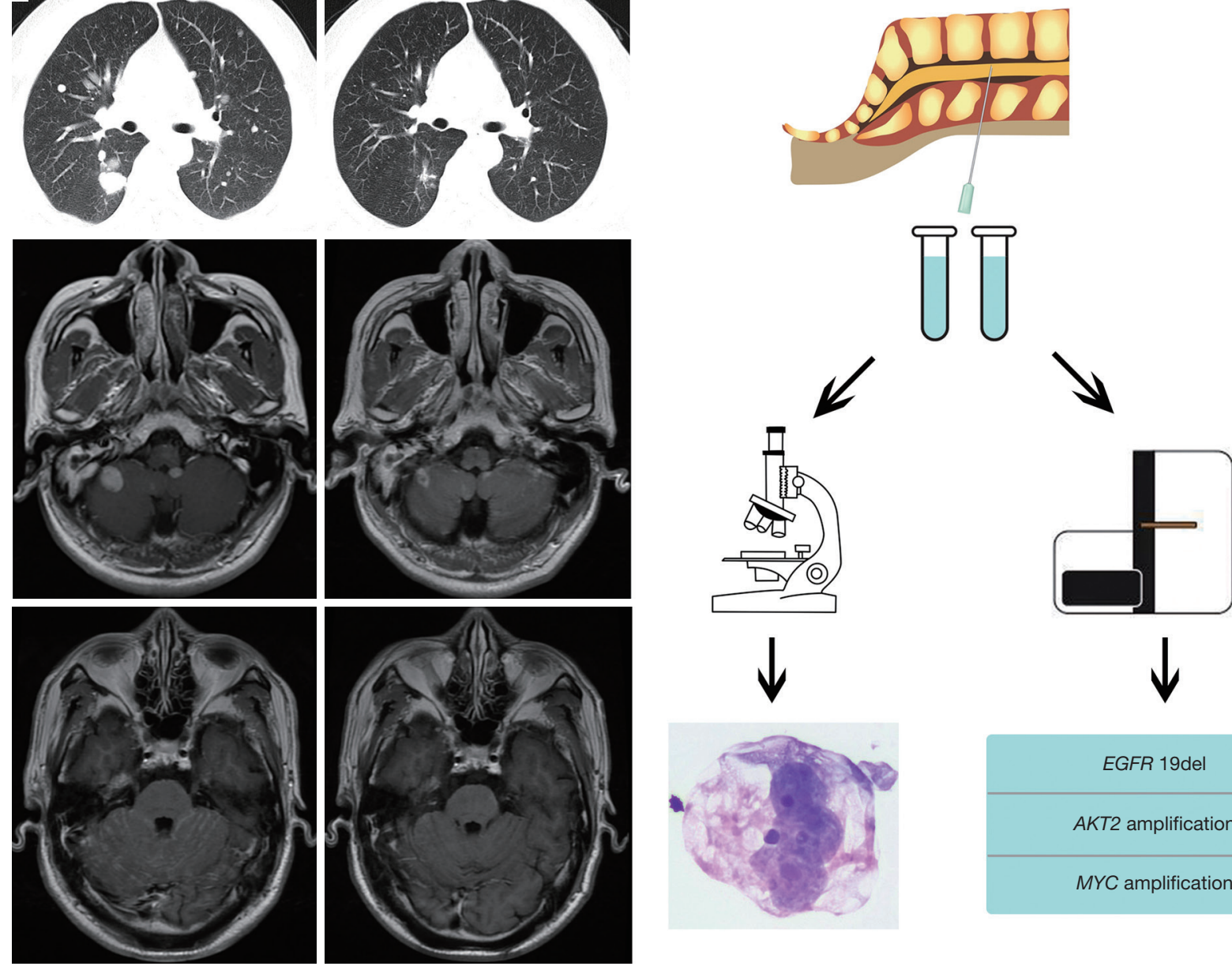

Baseline

After 6 weeks
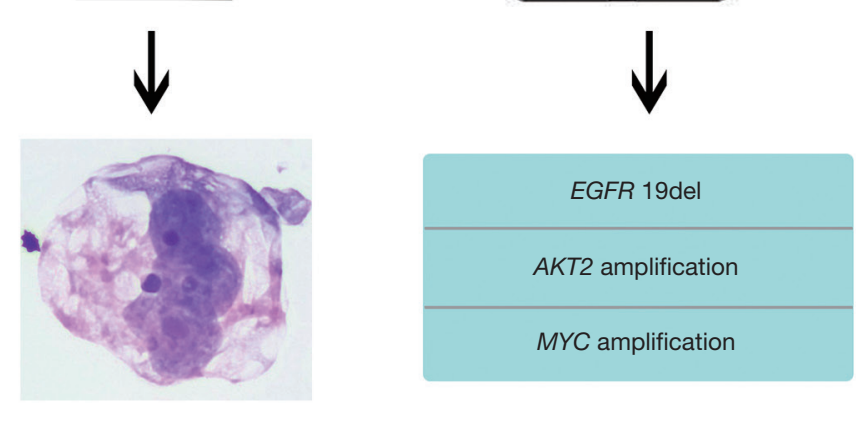

Figure 1 (A) Chest computed tomography and brain magnetic resonance imaging images before (at baseline) and after 6 weeks treatment of this case, which showed the excellent and durable response of both primary lesion, BMs and LM to this regimen; (B) flowchart of cerebrospinal fluid cytologic examination and genotype results of this case, which showed the leptomeningeal metastatic cells in cerebrospinal fluid with EGFR exon 19 deletion, AKT2 and MYC amplification.

was $55 \%$ according to RANO-LM radiologic criteria, a median LM PFS was 11.1 months, and a median OS was 18.8 months. Then, Yang et al. reported the BLOOM study (7), which demonstrated that osimertinib showed promising therapeutic efficacy in patients with EGFRmutated and LM (investigator-assessed ORR $41 \%$, PFS 8.6 months, OS 11.0 months). Recently, Lee et al. reported that patients with EGFR-mutated NSCLC with cytologically proven $\mathrm{LM}$ received osimertinib treatment had significantly better OS (4). The median OS was 17.0 months in osimertinib treatment group, which is comparable to the data in the BLOOM study. These results together indicate that osimertinib is effective and a promising therapeutic option for patients with EGFR-mutated NSCLC and LM. However, patients with LM had significantly shorter the median OS than those without or with asymptomatic CNS metastasis in the FLAURA study, suggesting that alternative treatment strategies are still needed.

Theoretically, the objective response of LM should be more sensitive to the CSF concentration of osimertinib because of metastatic tumor cells spreading to CSF, leptomeninges, and subarachnoid space. In the BLOOM study and a phase II trial $(7,8)$, double dose of osimertinib (160 mg once daily) showed striking efficacy in controlling LM. Furthermore, a recent study reported that $160 \mathrm{mg}$ of osimertinib could control the disease in six of eight 
patients who developed LM during $80 \mathrm{mg}$ of osimertinib therapy. Collectively, these findings suggest that $160 \mathrm{mg}$ of osimertinib could have better efficacy than $80 \mathrm{mg}$ in controlling LM. However, due to the limited sample size, further investigation to compare the efficacy of osimertinib 80 to $160 \mathrm{mg}$ in patients with LM is warranted.

The biologically synergistic antitumor activity of EGFR inhibition in combination with VEGF/VEGFR pathway blockade have been demonstrated in preclinical studies (9). Several recent phase $2 / 3$ trials have demonstrated that $E G F R$-TKIs plus bevacizumab could significantly improve PFS than EGFR-TKIs monotherapy in first-line treatment for patients with EGFR-mutated NSCLC $(10,11)$. Subgroup analysis reported that the addition of bevacizumab to EGFR-TKIs seem to have better PFS for those with CNS metastases (hazard ratios $=0.78$ ). A recent meta-analysis also indicated that patients with BMs at baseline in the EGFR-TKIs plus bevacizumab group had a trend toward better PFS (hazard ratios $=0.55, \mathrm{P}=0.001$ ) (12). Analogously, our previous publication indicated that EGFR-TKIs in combination with bevacizumab could significantly prolong both PFS and OS in those with EGFR-mutated NSCLC with multiple BMs (5). Moreover, a recent phase $1 / 2$ trial reported that osimertinib pus bevacizumab could be efficacious and protective against central nervous system progression (6). Here, we presented one case with EGFRmutated NSCLC and LM received osimertinib plus bevacizumab. Our results firstly suggested the excellent and durable response of both primary lesion, BMs and LM to this regimen. Notably, the phase II study of osimertinib plus bevacizumab for LM is already ongoing (NCT04425681). The result is anticipated. To date, we have at least three therapeutic choices, osimertinib $80 \mathrm{mg}$, osimertinib $160 \mathrm{mg}$ and osimertinib $80 \mathrm{mg}$ plus bevacizumab, for EGFRmutated NSCLC with LM. Which regimen is better in LM treatment need future investigations.

In conclusion, the current findings suggest that osimertinib or osimertinib in combination with bevacizumab could be considered as a promising treatment option for patients with central nervous system metastases, including BMs and LM. Future prospective and well-designed studies with large cohort are needed.

\section{Acknowledgments}

Funding: This study was supported by grants from Shanghai Municipal Key Clinical Specialty (shslczdzk02201), the National Natural Science Foundation of China (No.
81874036) and the Shanghai Sailing Program (No. 20YF1407500).

\section{Footnote}

Provenance and Peer Review: This article was a free submission to the journal. The article has undergone external peer review.

Peer Review File: Available at http://dx.doi.org/10.21037/ tlcr-20-984

Conflicts of Interest: All authors have completed the ICMJE uniform disclosure form (available at http://dx.doi. org/10.21037/tlcr-20-984). The authors have no conflicts of interest to declare.

Ethical Statement: The authors are accountable for all aspects of the work in ensuring that questions related to the accuracy or integrity of any part of the work are appropriately investigated and resolved.

Open Access Statement: This is an Open Access article distributed in accordance with the Creative Commons Attribution-NonCommercial-NoDerivs 4.0 International License (CC BY-NC-ND 4.0), which permits the noncommercial replication and distribution of the article with the strict proviso that no changes or edits are made and the original work is properly cited (including links to both the formal publication through the relevant DOI and the license). See: https://creativecommons.org/licenses/by-nc-nd/4.0/.

\section{References}

1. Alexander M, Lin E, Cheng H. Leptomeningeal Metastases in Non-small Cell Lung Cancer: Optimal Systemic Management in NSCLC With and Without Driver Mutations. Curr Treat Options Oncol 2020;21:72.

2. Ramalingam SS, Vansteenkiste J, Planchard D, et al. Overall Survival with Osimertinib in Untreated, EGFR-Mutated Advanced NSCLC. N Engl J Med 2020;382:41-50.

3. Jiang T, Su C, Ren S, et al. A consensus on the role of osimertinib in non-small cell lung cancer from the AME Lung Cancer Collaborative Group. J Thorac Dis 2018;10:3909-21.

4. Lee J, Choi Y, Han J, et al. Osimertinib Improves Overall Survival in Patients With EGFR-Mutated NSCLC With Leptomeningeal Metastases Regardless of T790M 
Mutational Status. J Thorac Oncol 2020;15:1758-66.

5. Jiang T, Zhang Y, Li X, et al. EGFR-TKIs plus bevacizumab demonstrated survival benefit than EGFRTKIs alone in patients with EGFR-mutant NSCLC and multiple brain metastases. Eur J Cancer 2019;121:98-108.

6. Yu HA, Schoenfeld AJ, Makhnin A, et al. Effect of Osimertinib and Bevacizumab on Progression-Free Survival for Patients With Metastatic EGFR-Mutant Lung Cancers: A Phase 1/2 Single-Group Open-Label Trial. JAMA Oncol 2020;6:1048-54.

7. Yang JCH, Kim SW, Kim DW, et al. Osimertinib in Patients With Epidermal Growth Factor Receptor Mutation-Positive Non-Small-Cell Lung Cancer and Leptomeningeal Metastases: The BLOOM Study. J Clin Oncol 2020;38:538-47.

8. Park S, Lee MH, Seong M, et al. A phase II, multicenter, two cohort study of $160 \mathrm{mg}$ osimertinib in EGFR T790Mpositive non-small-cell lung cancer patients with brain metastases or leptomeningeal disease who progressed on prior EGFR TKI therapy. Ann Oncol 2020;31:1397-404.

Cite this article as: Jiang T, Xu X, Chen X, Ding N, Hu Q, Zhou $\mathrm{C}, \mathrm{Hu}$ J. Osimertinib in combination with bevacizumab in EGFR-Mutated NSCLC with leptomeningeal metastases. Transl Lung Cancer Res 2020;9(6):2514-2517. doi: 10.21037/ tlcr-20-984
9. Schicher N, Paulitschke V, Swoboda A, et al. Erlotinib and bevacizumab have synergistic activity against melanoma. Clin Cancer Res 2009;15:3495-502.

10. Seto T, Kato T, Nishio M, et al. Erlotinib alone or with bevacizumab as first-line therapy in patients with advanced non-squamous non-small-cell lung cancer harbouring EGFR mutations (JO25567): an open-label, randomised, multicentre, phase 2 study. Lancet Oncol 2014;15:1236-44.

11. Saito H, Fukuhara T, Furuya N, et al. Erlotinib plus bevacizumab versus erlotinib alone in patients with EGFR-positive advanced non-squamous non-small-cell lung cancer (NEJ026): interim analysis of an open-label, randomised, multicentre, phase 3 trial. Lancet Oncol 2019;20:625-35.

12. Chen F, Chen N, Yu Y, et al. Efficacy and Safety of Epidermal Growth Factor Receptor (EGFR) Inhibitors Plus Antiangiogenic Agents as First-Line Treatments for Patients With Advanced EGFR-Mutated Non-small Cell Lung Cancer: A Meta-Analysis. Front Oncol 2020;10:904. 